\title{
Extraction of Ship Images using Deep Learning
}

\author{
Pavithra G.K., Shridevi. S
}

Abstract: Ship Extraction is very important in the marine industry. Extraction of ships is helpful to the fishers to find the other ships nearly around the particular area. Still today the fishers are to find the ships using some traditional methods. But now it became difficult due to environmental changes. So, by using the deep learning techniques like the CNN algorithm the ship extraction can be identified effectively. Generally, the ships are identified as narrow bow and parallel hull edge, etc. Here, the Existing system they have used the Tensor flow, to see the performance of the datasets, using Recall and precision. In the proposed system, we are using CNN deep learning techniques to identify the ships. By finding the ships with the techniques, the time will be saved and the productivity can be increased. The features of the ship image are taken and trained using the neural network algorithm and then the prediction is done by testing the images.

Keywords: Ship Extraction, Data augmentation, ResNet, CNN.

\section{INTRODUCTION}

The ship extraction from remotely sensed images has attracted much attention. It can supervise fishers and manage marine traffics to ensure its safety. With the developed of satellite and knowledge, automatic ship extraction has replaced the traditional manual ship detection, ships are extracting based on the shape and edge informations.

Eg: most of the ships under this caterogies narrow bow and parallel hull edge. So, which are easily defected because of the big difference between ships and water. Extraction of ship images using deep learning neural networks [1].Collecting several datasets based on vein recognition. Data sets organizations based on collected images. Implementing the system by use of certain algorithm techniques and feature extraction system [2]. Initially, the analysis is done whether the ship is there or not, then the classification is done to analyze the type of sea areas [3]. The analysis will be done using a feature extraction technique.

Which is time-consuming and also not more accurate. In this system, the marine engineers doesn't need to know the ship and no ship but applying the deep learning algorithm will find the ship accurately [4].

To predict whether the ships has a there or not the classification should be done [5]. For that, the two-step process should be followed. One is training which is the first step where we will have a large number of datasets for ship is there or not to train the machine. Testing is the second step where the current image is analyzed and predicted based on the training data [6].

Revised Manuscript Received on June 10, 2020.

* Correspondence Author

Pavithra G.K*, Vellore Institute of Technology, Chennai, India. Email: Pavithrag.k2015@vit.ac.in

Shridevi.S, Vellore Institute of Technology, Chennai, India. E-mail: shridevi.s@vit.ac.in.

(c) The Authors. Published by Blue Eyes Intelligence Engineering and Sciences Publication (BEIESP). This is an open access article under the CC BY-NC-ND license (http://creativecommons.org/licenses/by-nc-nd/4.0/)

\section{PROPOSED SYSTEM}

\section{INPUT IMAGE}

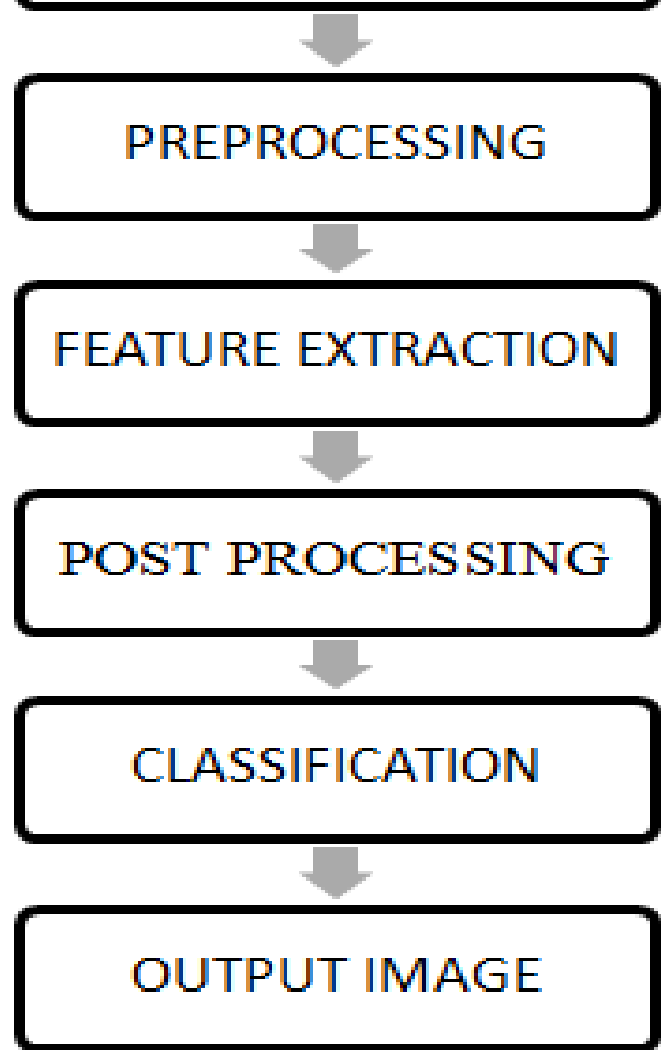

Figure 1 Architecture diagram of proposed System

The ship extraction system is considered as an image classification problem and can be performed using a deep learning algorithm. Hence the CNN architecture can be used to perform this ship extraction system. To perform CNN the required data should be collected using Data collection, data augmentation, pre-processing, and feature extraction as shown in fig 1. Normally the feature extraction is performed using features of the trained data and based upon that data, the threshold values can be kept and then in testing part the value of the features will compare with the trained one to predict the image the ship is there or not

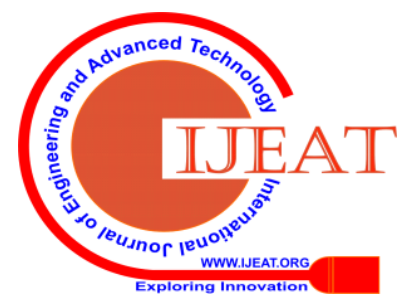




\section{Extraction of Ship Images using Deep Learning}

Flow chart diagram of the proposed System:

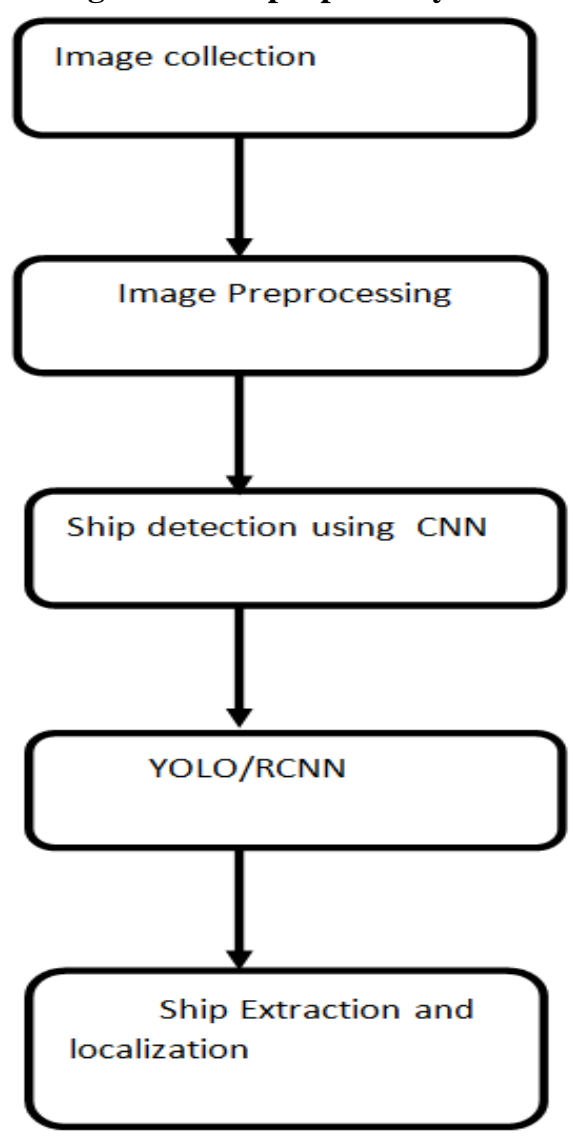

Figure 2 Flow Chart of Proposed System

First, the dataset is collected, then splits into two parts. $80 \%$ of training and $20 \%$ of testing. Totally we got 4000 images, here 3000 images or training data and it will consider as "no ship" or 0 and the balance 1000 images are consider as "ship" or 1 After that, By using the transfer learning technique the deep learning models are trained, and to indicate the significance of the model, their training plots are obtained. Then, for the classification of the images, performance metrics have been used, and finally, to detect and classify images, visualization techniques have been used. Basic CNN architecture and ResNet were implemented. The result of applying the filters to an input image will be capture by the feature maps of a CNN. Visualizing a feature map is to try to gain some understanding of the feature of CNN.

\section{MODEL DESCRIPTION}

\section{A. Data Collection}

The data collection deals with collecting the ship images. The images will be collected and filter according to the dimension, size, and format. The total data that are collected will be stored in a database in one format.

\section{B. Data Augmentation}

Data augmentation is normally performed to change the dimension or direction of an image. Normally the 3-pixel images are in the vertical direction and 5-pixels are in the horizontal direction.

Normally the data augmentation method is used to find the ships from the sea of an image, and then the horizontal flipping to achieve the neural network architecture.

\section{Image Preprocessing}

The elimination of the noise in the picture to adjust the pixel values is called preprocessing.

\section{Feature Extraction}

It's an important part to gracefully predict the ship felt region. Shape and textual extraction are done here. To determine the finding the each ship, the ship image is captured and processed.

\section{CNN model:}

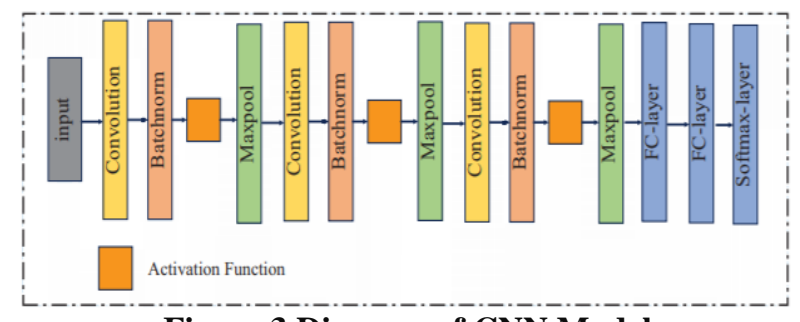

Figure 3 Diagram of CNN Model

The pre-trained CNN model as shown fig 3. The CNN Model can be treated as a feature extractor. A linear classifier can be built by using extracted features as input. On the other hand, the fine-tuning method is often carried out to fine-tune some high-level layers. Features in the early layers are more generic. While features in the later layers contain more specific information of original datasets.

Freezing early layers can bring us general and useful features for many tasks. And fine-tuning following layers can generate more particular features existing in our datasets.

ResNet incorporates identify shortcut connections which essentially skip the training of one or more layers - creating a residual block. This model gives $99 \%$ of accuracy on our dataset.

ResNet model (Transfer learning):
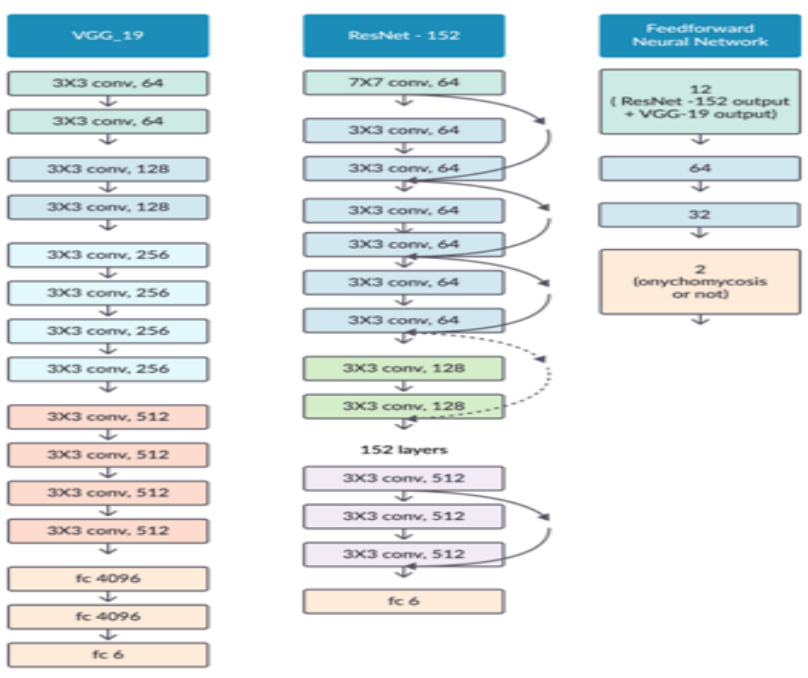

Figure 4 Diagram of ResNet Model

The ResNet Model as shown fig 4.

In CNN architecture while training at certain condition adding the neural network will slow down the training process and also saturate the accuracy. ResNet model gives $66 \%$ of accuracy. Often carried out to fine-tune some high-level layers.

Published By:

Blue Eyes Intelligence Engineering

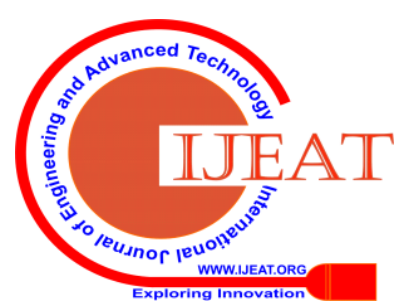


Features in the early layers are more generic. While features in the later layers contain more specific information of original datasets.

\section{RESULT AND DISCUSSION}

With the 4000 images, The proposed system has got an accuracy of $99 \%$ and $66 \%$ by using algorithms CNN and ResNet respectively, Which is comparatively higher than the existing system.

\section{Output Snaps:}

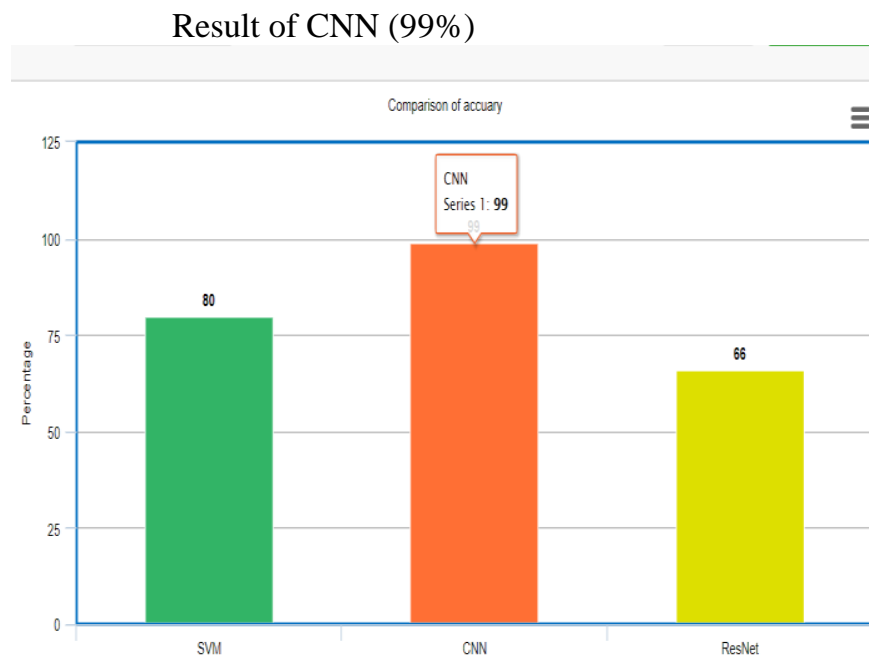

Figure 5 Comparison of Outputs

\section{Graphs:}

For CNN:

Loss of Testing and Training;

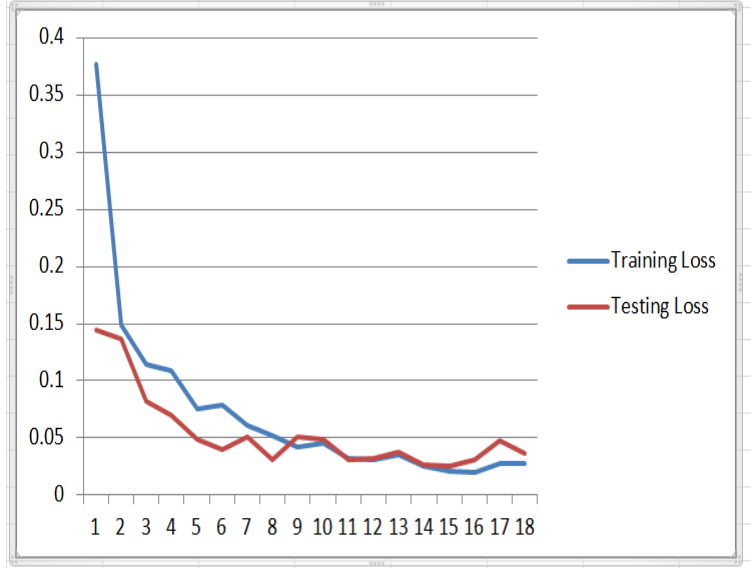

Figure 6 Diagram of Loss Comparison

Accuracy of Testing and Training:

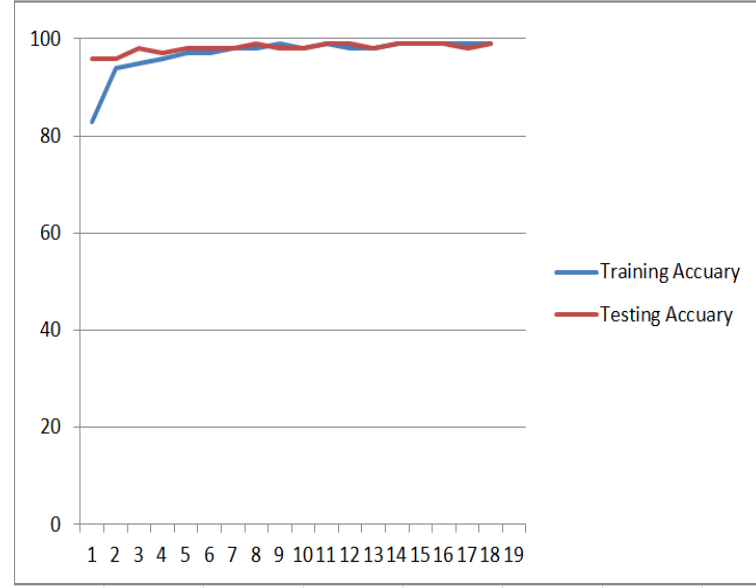

Figure 7 Diagram of Accuracy Comparison Comparison Table of Results:

\begin{tabular}{|c|c|c|c|c|}
\hline \multirow[t]{2}{*}{ S.NO } & \multicolumn{2}{|c|}{ Existing System } & \multicolumn{2}{|c|}{ Proposed System } \\
\hline & Algorithm & Pelcentage & Algorithm & Percentage \\
\hline 1. & CNN & 94 & CNN & 99 \\
\hline 2 & & & ResNlet & 66 \\
\hline
\end{tabular}

Figure 8 Table of Algorithm Results

\section{CONCLUSION}

Deep learning technologies has become one of the important technologies in the marine industry. This paper came with an idea of identifying the ships in the sea. So that we can identify the ships easily and it will helpful to the marine industry. The infected segment of the leaf is analyzed by the CNN architectures. It will be very useful for the marine industry especially in oceans. To finding the ships and to extract the particular area for the feature extracting technique was very helpful.

\section{FUTURE WORK}

The future enhancement is to develop the open multimedia (Audio/ video) about the ships, Once the ship is extracted.

\section{REFERENCE}

1. K. Eldhuset, "An automatic ship and ship wake detection system for space borne SAR images in coastal regions".

2. X. Xing, K. Ji, L. Kang, and M. Zhan, "Review of ship surveillance technologies based on high-resolution wide-swath synthetic aperture radar imaging".

3. C. C. Wackerman, K. S. Friedman, W. G. Pichel, P. Clemente-Colón, and $\mathrm{X}$. Li, "Automatic detection of ships in RADARSAT-1 SAR imagery".

4. C. R. Zhu, H. Zhou, R. S. Wang, and J. Guo, "A Novel Hierarchical Method of Ship Detection from Spaceborne Optical Image Based on Shape and Texture Features".

Published By:

Blue Eyes Intelligence Engineering

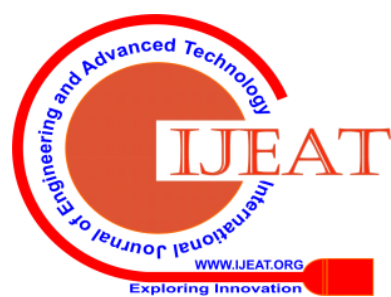




\section{Extraction of Ship Images using Deep Learning}

5. J. X. Tang, C. W. Deng, G. B. Huang, and B. J. Zhao, "Compressed Domain Ship Detection on Spaceborne Optical Image Using Deep Neural Network and Extreme Learning Machine".

6. R. Zhang, J. Yao, K. Zhang, C. Feng, and J. Zhang, "S-CNN-based ship detection from high-resolution remote sensing images," presented at the International Archives of the Photogrammetry, Remote Sensing Spatial Information Sciences Prague, Czech Republic, July, 2016.

7. G. Liu, Y. Zhang, X. Zheng, X. Sun, K. Fu, and H. Wang, "A new method on inshore ship detection in high-resolution satellite images using shape and context information."

8. J. Xu, X. Sun, D. Zhang, and K. Fu, "Automatic detection of inshore ships in high-resolution remote sensing images using robust invariant generalized Hough transform."

9. L. Huo, L. Jiao, S. Wang, and S. Yang, "Object-level saliency detection with color attributes," Pattern Recognition, vol. 49, pp. 162173, 2016.

10. C. Dong, J. Liu, and F. Xu, "Ship Detection in Optical Remote Sensing Images Based on Saliency and a Rotation-Invariant Descriptor."

11. Q. P. Li, L. C. Mou, Q. J. Liu, Y. H. Wang, and X. X. Zhu, "HSF-Net: Multiscale Deep Feature Embedding for Ship Detection in Optical Remote Sensing Imagery".

12. Y. Yao, Z. G. Jiang, H. P. Zhang, D. P. Zhao, and B. W. Cai, "Ship detection in optical remote sensing images based on deep convolutional neural networks."

https://www.kaggle.com/rhammell/ships-in-satellite-imagery.

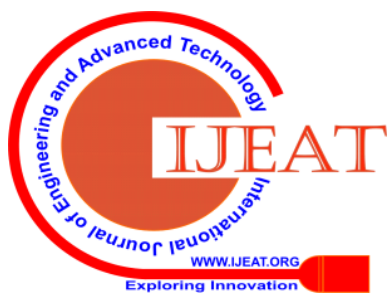

